Research Article

\title{
Theoretical Analysis of a Novel WDM Optical Long-Haul Network Using the Split-Step Fourier Method
}

\author{
Mounia Chakkour (D), Otman Aghzout $\mathbb{D}$, and Fahd Chaoui \\ Department of Computer Science Engineering, SIGLab, National School of Applied Sciences, Tetuan, Morocco \\ Correspondence should be addressed to Otman Aghzout; oaghzout@uae.ac.ma
}

Received 19 June 2019; Revised 29 January 2020; Accepted 1 February 2020; Published 1 March 2020

Academic Editor: Giulio Cerullo

Copyright (C) 2020 Mounia Chakkour et al. This is an open access article distributed under the Creative Commons Attribution License, which permits unrestricted use, distribution, and reproduction in any medium, provided the original work is properly cited.

In this paper, a new optical transmission model was investigated and developed in order to improve the performance of Wavelength Division Multiplexing optical transmission systems. For this purpose, an efficient theoretical method combining a Fiber Bragg Grating (FBG) and Erbium Doped Fiber Amplifier (EDFA) was implemented. Enhancements on the transmitted signal amplitudes with important compensation of the chromatic dispersions and large reduction on the nonlinear effects were obtained. The reflectivity peak, the full width at half maximum bandwidth, and the side lobe level have been studied in depth to demonstrate the efficiency of the proposed method. To apply the new method, we considered a complicated system of 16-channel wavelengths, where the split-step fourier method was used to solve the nonlinear Schrödinger equations, and the transfer matrix method was applied to describe the coupled mode equations. The wavelengths corresponding to different values of reflectivity peaks calculated previously in the FBG outputs section were injected as EDFA inputs to determine the output power and the gain of the new transmission system. All results are analyzed using Matlab program.

\section{Introduction}

In recent years, Wavelength Division Multiplexing communication systems have been widely used as a solution to the increasing demand of high bit rate speed networks, which employ optical fiber as a medium for telecommunication and networking [1]. WDM is basically an optical transmission technique, which multiplexes different signals of different wavelength and can provide data capacity beyond hundreds of Gigabit per second over thousands of kilometers in a single mode fiber. However, in long-haul high-speed WDM systems, the signal transmission is limited by different impairments, such as fiber losses and signal attenuation [2-4]. For this reason, new technologies come emerge to meet the new challenges of WDM technology development and the related growth of requirements on transmission capacity of optical communication systems. Optical amplifiers have been commonly deployed in transcontinental, submarine, and other state-of-the-art long distance connections to help in extending power budget, thus reducing the number of connectivity points. Optical amplifiers are also designed to lessen the effects of dispersion and attenuation, which allows for improved performance for long-haul optical systems. There are different types of amplifiers such as Fiber Raman Amplifier (FRA) and Erbium Doped Fiber Amplifier (EDFA) that have been introduced to encounter the new WDM challenges. Raman amplifiers improve the noise figure and reduce the nonlinear penalty of fiber systems, and this improves the overall system performance thus allowing longer amplifier spans, higher bit rates, and closer channel spacing [5]. Another option for amplifications is EDFA, which results in an overall superior performance due to low loss optical window of silica-based fiber. EDFA also has large gain bandwidth, which is normally tens of nanometers, and it is adequate to amplify data channels with the highest data rates without presenting any effects of gain narrowing $[6,7]$. Nevertheless, the use of optical amplification has generated new problems such as group velocity dispersion and nonlinear fiber effects. FourWave Mixing (FWM), Stimulated Raman Scattering (SRS), 
and Cross-Phase Modulation (XPM) are the most dominant types of nonlinear effects that have been generated due to the optical amplification and limit the performance of optical transmissions [8-10]. Many numerical methods have been proposed to compensate the chromatic dispersion and reduce the nonlinear effects in optical fiber transmission systems $[4,11,12]$. In this work, we propose a new optical transmission model to improve the performance of WDM optical transmission systems using Fiber Bragg Grating (FBG) and EDFA based on the Split-Step Fourier Method (SSFM). For this purpose, a new code has been developed to simulate in details and evaluate the performance of optical WDM transmission system of 16 channels in terms of nonlinear and dispersion parameters. Firstly, the SSFM has been applied to solve the nonlinear Schrödinger equation that describes the light propagation in optical fibers. The TTM has been also applied to simulate the reflected spectrum of Fiber Bragg Grating Sensor of 16 channel WDM network with the aim to calculate the reflectivity peak, Full Width at Half Maximum (FWHM), and the Side Lobe Level (SLL). Finally, the wavelengths corresponding to different values of reflectivity peaks calculated previously in the FBG output were used as EDFA inputs to determine the output power and the gain of the transmission system. The optical transmission system under study has been compared with a similar work published previously by other authors [13]. Results obtained from the study in the paper demonstrate a very high efficiency of the developed method.

\section{WDM Transmission System Using FBG and EDFA}

2.1. Design Consideration. The optical transmission systems have undergone enormous upgrades due to large capacity demand for data transmission. However, different factors still adversely affect the optical transmission systems like the chromatic dispersion, the attenuation phenomena, and the nonlinear effects [14]. Many ideas were proposed by authors to find solutions to these problems. But, in many situations, these kind of problems still remain $[5,15]$. Therefore, the main objective of this paper is to propose a new method to face these challenges and obtain results with high efficiency. For this purpose, a simple theoretical method based on the combination of FBG and EDFA will be integrated in the same transmission system, as shown in Figure 1.

Figure 1 shows the optical system designed in this paper. The EDFA is introduced with the aim to encounter the attenuation phenomena, while the FBG is introduced to compensate chromatic dispersion and to decrease nonlinear effects [16]. To determine the gain of the optical network, we have divided the simulation system into three sections, the first one is formed by the multiplexing output with singlemode fiber (SMF), the second is made up by SMF-FBG with FBG-EDFA, and the third section is formed by EDFA with the demultiplexing input. During the application of this method, a new problem has been encountered, viz., the manner in which the FBG can be combined with the EDFA. $\mathrm{We}$, therefore, propose in Figure 2 the most convenient method to solve this problem. This method consists of

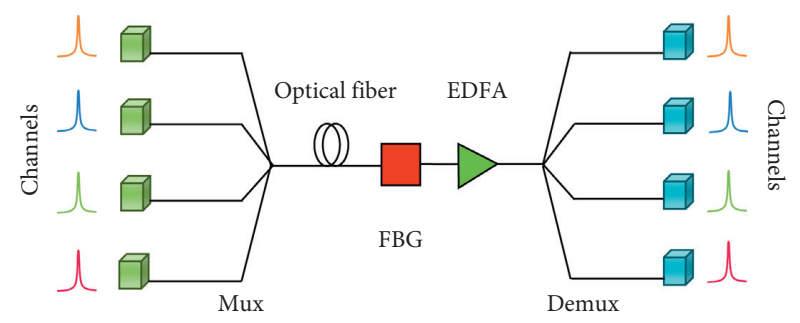

Figure 1: Optical transmission system design.

injecting the wavelengths corresponding to different values of reflectivity peaks calculated previously in the FBG outputs section as EDFA inputs. Then, the output wavelengths of the FBG section will be considered as input for EDFA. This solution can be considered as the novelty of our work.

2.2. Method Description. In order to obtain a very high efficiency of the system responses, the developed method has been carefully computed to provide results with high precision. As a first step, the well-known SSFM was used to assess the performance of the developed model in terms of nonlinear and dispersion parameters [8]. We have applied SSFM to solve the nonlinear partial differential equations such as nonlinear Schrödinger equations (NLSE) [13]. As known, SSFM is a pseudospectral numerical method commonly used to analyze the nonlinear effects in optical fiber. The solution to the nonlinear partial differential equations is given as follows:

$$
\begin{aligned}
\frac{\partial A}{\partial z}= & \left(-\frac{\alpha}{2}-i \frac{\beta_{2}}{2} \frac{\partial^{2}}{\partial T^{2}}+\frac{\beta_{3}}{6} \frac{\partial^{3}}{\partial T^{3}}\right) A \\
& +i \gamma\left(|A|^{2}+i S \frac{1}{A} \frac{\partial}{\partial T}\left(A|A|^{2}\right)-T_{R} \frac{\partial|A|^{2}}{\partial T}\right) A, \\
\frac{\partial A}{\partial z}= & D(A)+N(A),
\end{aligned}
$$

where $D(A)$ is the operator of all linear terms in $A$ and $N(A)$ is the operator of all nonlinearities. By using these definitions, equation (3) can be expressed briefly as

$$
\frac{\partial A}{\partial z}=(\mathscr{D}+\mathcal{N}) A .
$$

If we assume that $\mathscr{D}$ and $\mathcal{N}$ are independent of $z$, equation (4) can be integrated exactly and the solution at $z+h$ will be

$$
A(z+h, T)=\exp (h(\mathscr{D}+\mathcal{N})) A(z, T) .
$$

For a commonly used symmetric approximation [8], equation (5) is written as follows:

$$
A(z+h, T)=\exp \left(\frac{h}{2} \mathscr{D}\right) \exp (h \mathcal{N}) \exp \left(\frac{h}{2} \mathscr{D}\right) A(z, T) .
$$

In the second section, we will solve the coupled mode theory equations with the aim to obtain the spectrum 


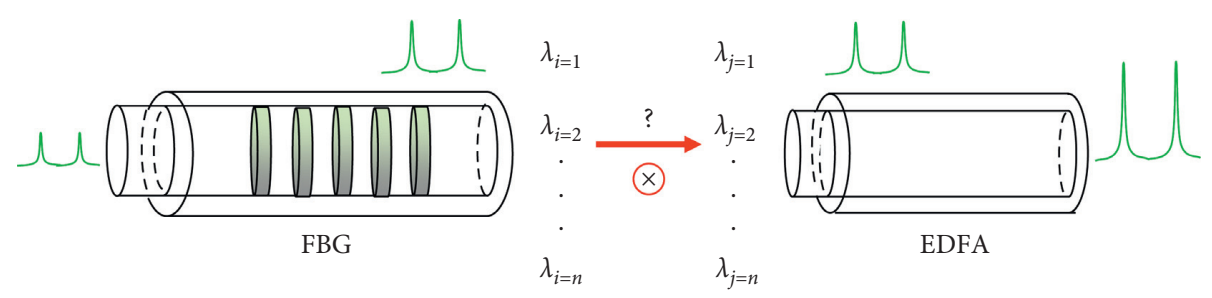

FIgURE 2: Optical transmission system configuration.

responses. In this section, the TMM is applied to get the optimized reflection spectrum based on Reflectivity, SSL, and FWHM of each wavelength separately. Therefore, a new algorithm describing the theoretical model has been detailed. To determine the output power and the gain of the whole optical transmission network, one of the toughest challenges encountered during the construction of the new algorithm is how to link the FBG and the EDFA sections. As a solution, firstly, we have calculated the wavelength matrix from the first section corresponding to the different values of the reflectivity peaks calculated previously as EDFA inputs. In short, the outputs of FBG sections are considered as the inputs of the EDFA sections.

A detailed algorithm developed in this paper which illustrates the main steps followed to assess and enhance the transmission optical network performance is presented in Figure 3. The flowchart of this algorithm has been carefully programmed to examine the chromatic dispersion and the nonlinear effects. The optical network properties are introduced as the first step. Then, the SSFM method is applied to solve the NLSE equations with the aim to evaluate the performance of the developed model in terms of nonlinear and dispersion parameters. Coupling Mode Theory and TMM have been introduced to obtain the spectral response of the FBG based on our new code with the aim to obtain the three critical parameters, which are Reflectivity level, FWHM, and SLL. The different wavelengths corresponding to different values of reflectivity peaks in the FBG outputs obtained previously have been used as EDFA inputs, in order to determine the output power and the gain of the transmission optical network.

\section{Results and Discussions}

In the first part, a new method will be developed to study the performance of an optical transmission system of 16 channels in order to determine the gain of the optical transmission network in terms of nonlinear and dispersion parameters.

\subsection{Output Reading of the System without EDFA and FBG.} To understand the manner in which the FBG and EDFA affect the communications in Figure 1, as a first step, the optical transmission system has been studied and investigated without these two components as shown in Figure 4 with the aim to evaluate the performance of the developed method in this paper.

3.1.1. Output Signal Amplitudes. Figure 5 represents the 3D behavior of the output signal amplitudes for different values of optical fiber without the FBG and EDFA after solving Schrodinger equations in the time domain. It can clearly be observerd from Figure 5 that when the input power increases, the value of amplitude also increases. Figure 6 describes the output signal amplitudes of optical transmission fiber versus time in SSFM application for numerical modeling results.

We can obviously observe that the input power of $3 \mathrm{mw}$ has the highest amplitude value, and the input power of $1 \mathrm{mw}$ has the lowest amplitude value. It can be clearly also noticed that when the input power increases, the ideal spectrum of the output signal amplitudes was achieved.

3.1.2. Dispersion Length and Nonlinear Length. In order to study the optical transmission system performance without the FBG and the EDFA in terms of the length dispersion $L_{\mathrm{D}}$ and the length nonlinear $L_{\mathrm{NL}}$, the SSFM method was applied. The expression of the dispersion length is simplified as

$$
L_{\mathrm{D}}=\frac{T_{0}^{2}}{\left|\beta_{2}\right|} \text {. }
$$

The nonlinear length can be determined from this relationship.

$$
L_{\mathrm{LN}}=\frac{1}{\gamma P_{0}},
$$

where $T_{0}$ is the input pulse width, $\beta_{2}$ is the dispersion of the group velocity, $P_{0}$ is the peak power, and $\gamma$ is the nonlinearity coefficient.

Figure 7 presents the variation of dispersion length $L_{\mathrm{D}}$ and nonlinear length $L_{\mathrm{NL}}$ at different values of wavelengths with $L=100 \mathrm{~km}$.

As can be seen from Figure 7, when the wavelength increases, the nonlinear length increases proportionally, while the value of the dispersion length remains almost the same. It can also be noticed that the fiber length is higher than the nonlinear length and the dispersion length, e.g., $L>L_{\mathrm{D}}$ and $L>L_{\mathrm{NL}}$. We can conclude that, when the fiber length $L$ is longer or comparable to $L_{\mathrm{D}}$ and $L_{\mathrm{NL}}$, dispersion and nonlinearity dominate as the pulse propagates along the fiber.

3.1.3. Gain and the Output Power across the Optical Transmission System. Table 1 presents the output power and the gain of each channel of the optical network without the EDFA and the FBG. It can be seen that the values of output power and gain increases gradually as the wavelength increases. The expression of the output power is simplified as 


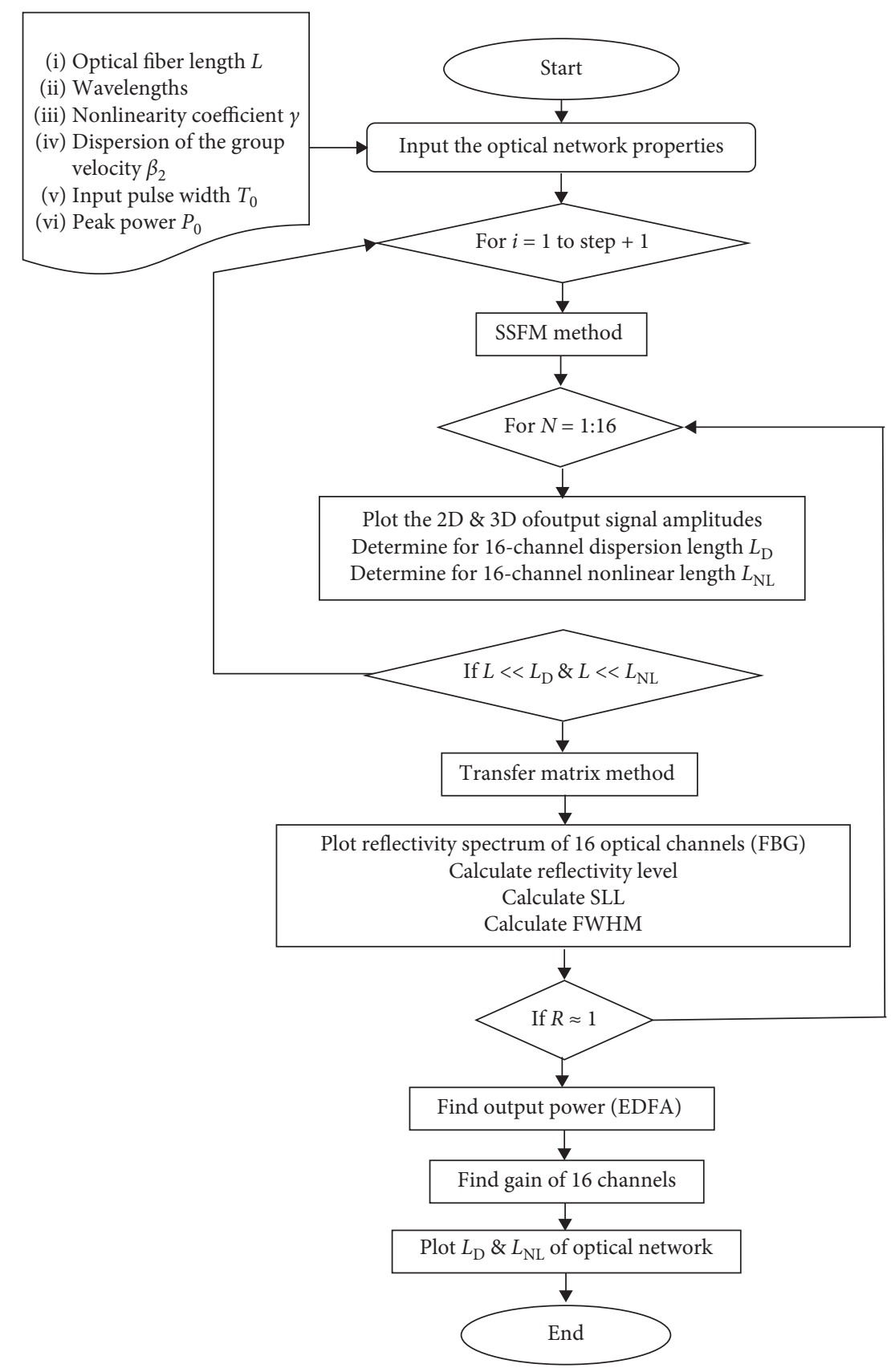

FIGURE 3: Flowchart employed in this study.

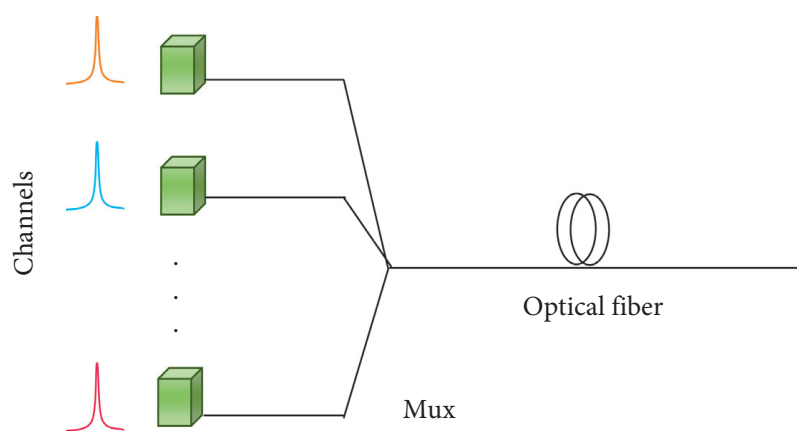

Figure 4: Optical transmission system without FBG and EDFA components. 


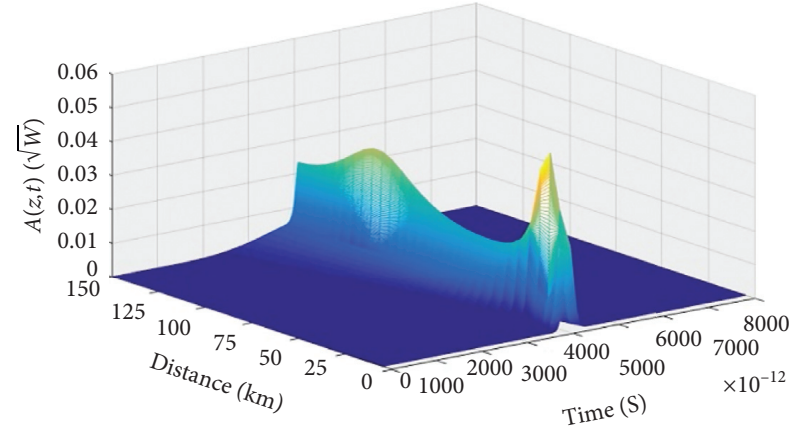

(a)

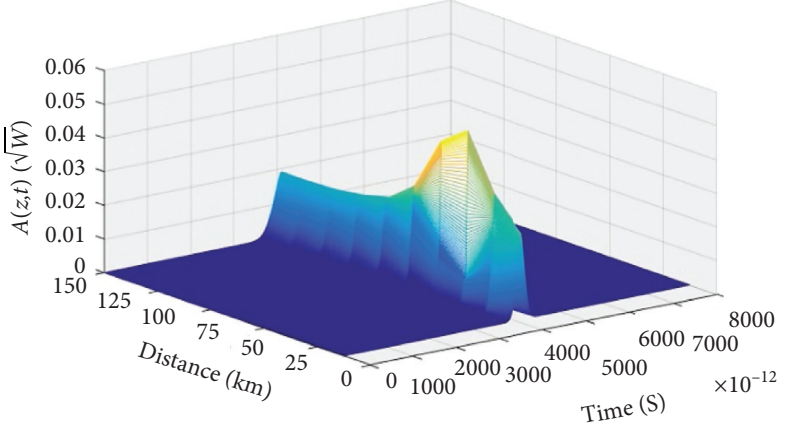

(b)

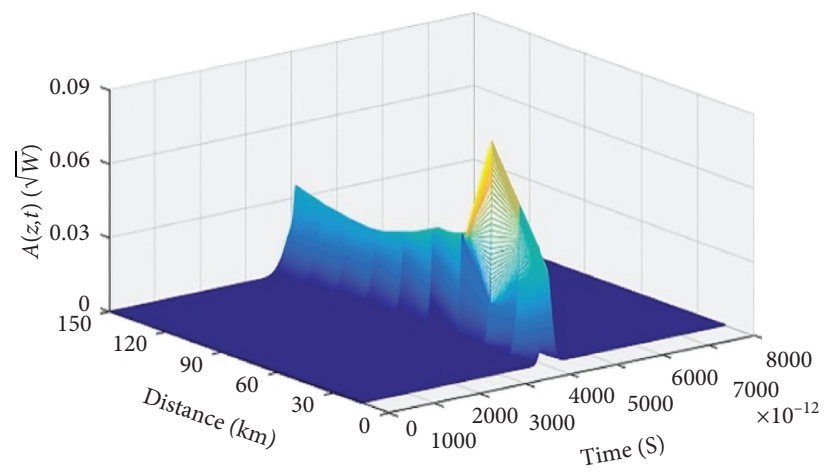

(c)

Figure 5: Output signal amplitudes of optical fiber for different values of length (a) Pin $=1 \mathrm{~mW}$. (b) Pin $=2 \mathrm{~mW}$. (c) Pin $=3 \mathrm{~mW}$.

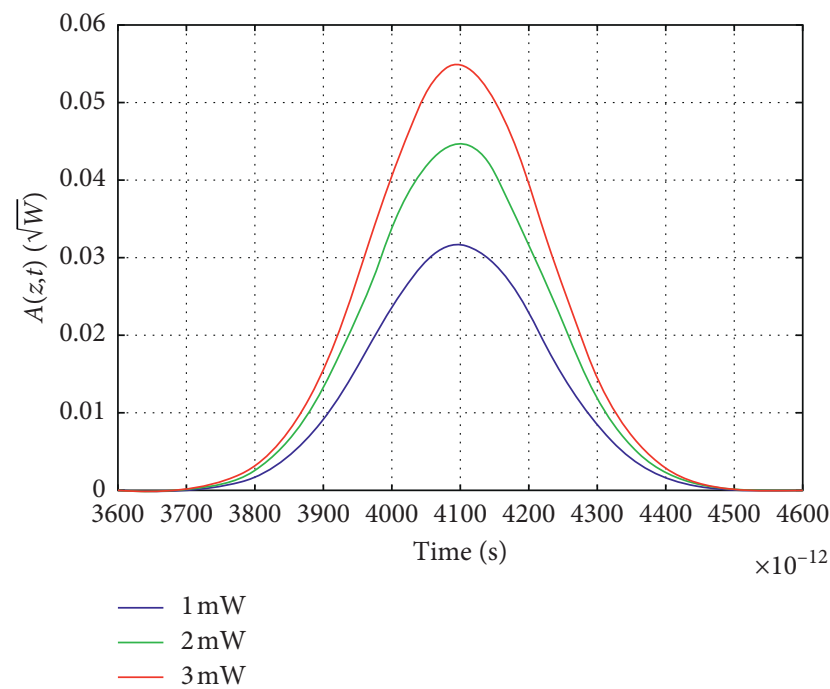

Figure 6: Output signal amplitudes for different values of input power.

$$
P_{\text {out }}=10 \log _{10}\left(\frac{P_{\mathrm{sp}}}{1 e^{-3}}\right) \text {, }
$$

where $P_{\mathrm{sp}}$ is the power signal in $z+$. The gain can be determined from this relationship by

$$
G=\frac{P_{\text {out }}}{P_{\text {in }}} .
$$

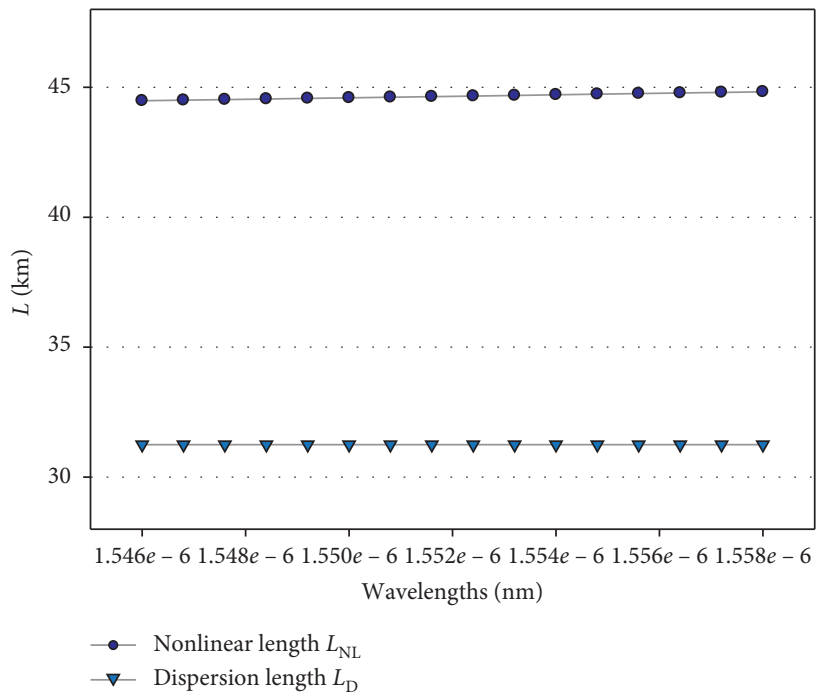

FIGURE 7: Variation of $L_{\mathrm{D}}$ and $L_{\mathrm{NL}}$ at different values of wavelengths in $100 \mathrm{~km}$.

3.2. Output Reading of the New Method with EDFA and FBG. In this section, we study the optical system presented in Figure 1. As can be seen from the figure, two new additional components (FBG and EDFA) have been integrated in the design to enhance the responses in terms of the chromatic dispersion, the attenuation phenomena, and the nonlinear effects such as the FWM. The optical transmission system has been studied in detail, and all parameter effects were 
TABLE 1: Output readings of optical network.

\begin{tabular}{lcc}
\hline Wavelengths $(\mathrm{nm})$ & Gain $(\mathrm{dB})$ & Output power $(\mathrm{dBm})$ \\
\hline 1546 & 32.45 & 09.94 \\
1546.8 & 32.80 & 09.96 \\
1547.6 & 33.15 & 09.98 \\
1548.4 & 33.49 & 10.00 \\
1549.2 & 33.84 & 10.02 \\
1550 & 34.19 & 10.04 \\
1550.8 & 34.54 & 10.06 \\
1551.6 & 34.89 & 10.08 \\
1552.4 & 35.24 & 10.10 \\
1553.2 & 35.59 & 10.12 \\
1554 & 35.94 & 10.14 \\
1554.8 & 36.29 & 10.15 \\
1555.6 & 36.64 & 10.17 \\
1556.4 & 36.99 & 10.19 \\
1557.2 & 37.34 & 10.21 \\
1558 & 37.69 & 10.25 \\
\hline
\end{tabular}

taken into account to obtain efficient results. The comparison results of this new model and the similar one without FBG and EDFA will be presented posteriorly in terms of LD, LNL, the output signal amplitude, the gain, and the output power.

Initially, the dispersion length and the nonlinear length were studied. In Figure 8, we present the variation of nonlinear length at different values of wavelengths. Results prove that, the system with FBG and EDFA offers higher $L_{\mathrm{NL}}$ values in comparison with the same one without these two components. Based on these results, we can conclude that the implementation of FBG and EDFA in the communication design of Figure 1 can surely reduce significantly the nonlinear effects.

Figure 9 presents the chromatic dispersion compensation effects of the proposed model, and it can be observed that the developed method offers better performance as the length of dispersion without FBG and EDFA is much lower compared to when FBG and EDFA are used (almost $500 \mathrm{~km}$ ). When fiber length $L$ is such that $L<<L_{\mathrm{NL}}$ and $L<<L_{\mathrm{D}}$, neither dispersive nor nonlinear effects play a significant role during pulse propagation.

3.2.1. Output Signal Amplitudes. The output signal amplitudes of the proposed method without and with the FBG and the EDFA for different optical fiber length values are depicted in Figure 10. It can be clearly seen that the use of the suggested method offers better output signal amplitudes for long optical fiber length values.

To demonstrate the efficiency of the new developed method, the simulation results of the developed model have been compared with (11 s). The result comparison considers the same initial settings: the fiber length at $50 \mathrm{~km}$ and the input power is $1 \mathrm{~mW}$.

Figure 11 shows the comparison results of maximal values of output signal amplitudes in dependency of optical fiber length, it can obviously be observed that our proposed model offers better amplitude for all values of fiber length, which proves the efficiency of the developed optical network

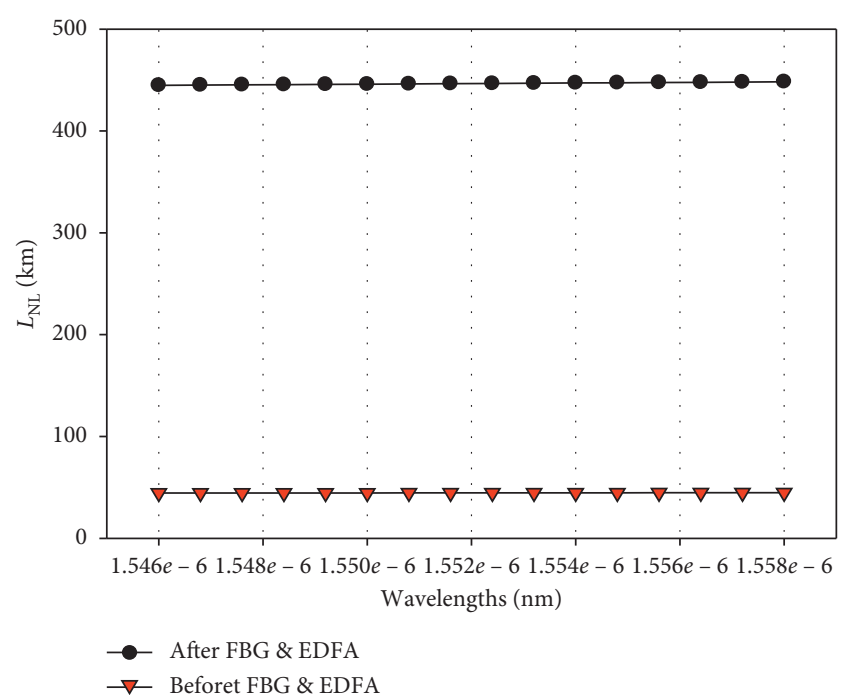

Figure 8: Variation of $L_{\mathrm{NL}}$ at different values of wavelengths.

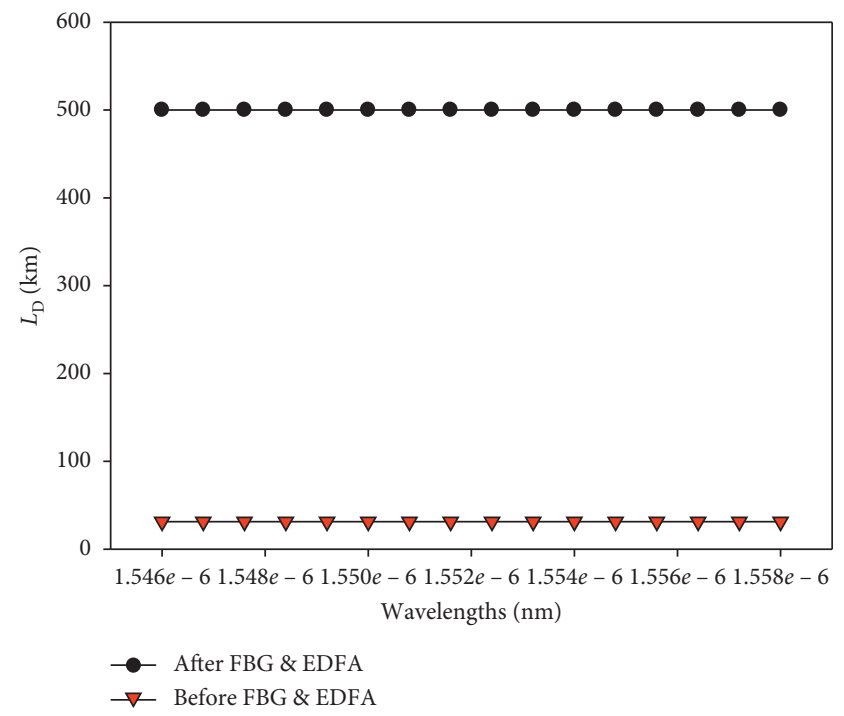

Figure 9: Variation of $L_{\mathrm{D}}$ at different values of wavelengths.

to reduce nonlinear effects and compensate chromatic dispersion in WDM optical transmission systems.

3.2.2. Reflectivity Spectrum for 16 Optical Channels WDM. To get the optimized reflection spectrum of the developed model, the Coupled Mode Theory (CMT) and the TMM were applied. For this purpose, a new code has been developed to determine the reflectivity peak, SSL, and FWHM of each wavelength separately.

The use of WDM technology in optical transmission systems and their related optical networks affects the performance of optical systems. However, it can be clearly noticed from Figures 12 and 13 that, in the proposed model, the 16 transmitted wavelengths keep a high reflectivity with a low side lobe level and narrow FWHM, which further demonstrates the effectiveness of the proposed model [11]. 


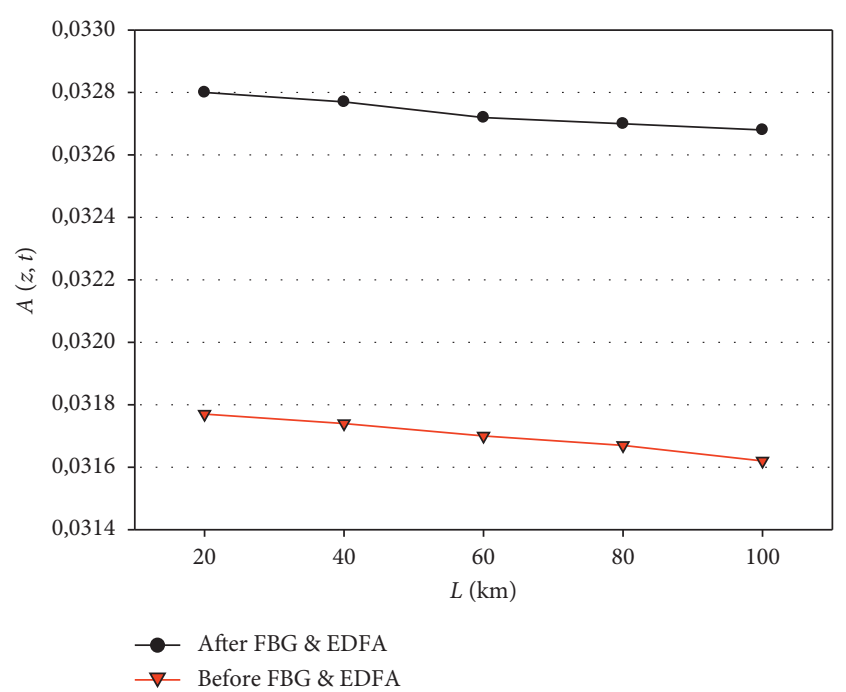

FIGURE 10: Output signal amplitudes variation at different values of optical fiber length.

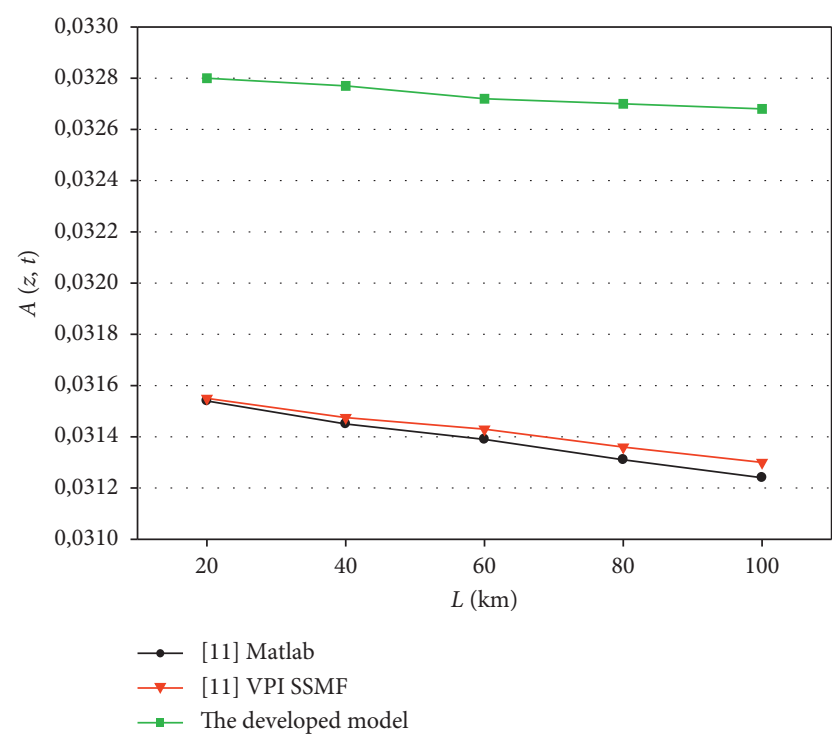

Figure 11: Variation of output signal amplitudes variation at different values of optical fiber length.

Table 2 represents the results of the output readings at different values of wavelengths. It can be obviously observed that a high reflectivity level, an efficient side lobe suppression, and small FWHM have been achieved in this study.

\subsubsection{Output Power and Gain for 16 Optical Channels WDM.} The second section of the optical transmission system design is formed by SMF-FBG with FBG-EDFA. The corresponding wavelength of reflectivity peaks are shown in Table 3.

Figure 14 represents the output power of the developed model with FBG and EDFA, and without FBG and EDFA. It can be observed that the developed method with FBG and EDFA gives better output power with high performance all over wavelengths.

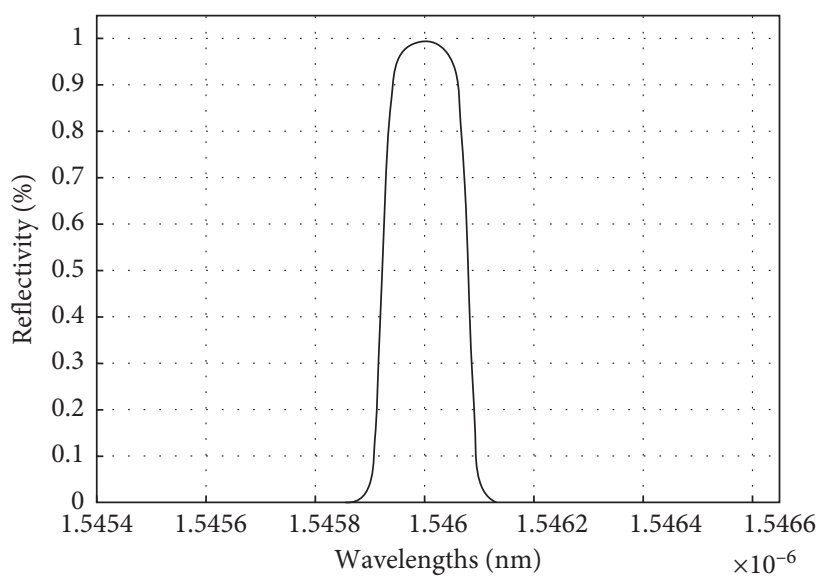

FIGURE 12: Reflectivity of one optical channel.

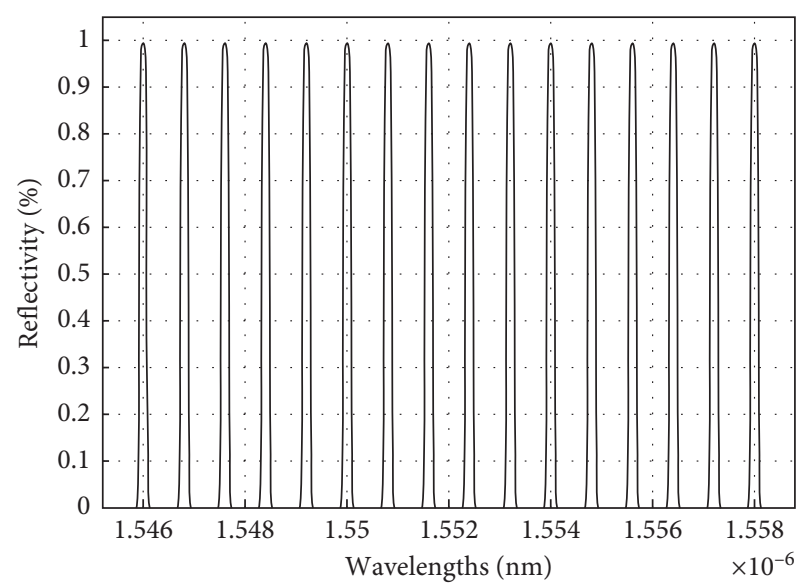

FIGURE 13: Reflectivity of 16 optical channels.

TABLe 2: Reflectivity, FWHM, and SLL of 16 wavelengths.

\begin{tabular}{lccc}
\hline Wavelength $(\mathrm{nm})$ & Reflectivity $(\%)$ & FWHM $(\mathrm{m})$ & SLL $(\mathrm{dB})$ \\
\hline 1546 & 99.19 & $1.5459 e-10$ & -32.6672 \\
1546.8 & 99.19 & $1.5467 e-10$ & -32.6696 \\
1547.6 & 99.18 & $1.5475 e-10$ & -32.6724 \\
1548.4 & 99.18 & $1.5483 e-10$ & -32.6758 \\
1549.2 & 99.18 & $1.5491 e-10$ & -32.6795 \\
1550 & 99.17 & $1.5499 e-10$ & -32.6838 \\
1550.8 & 99.17 & $1.5507 e-10$ & -32.6885 \\
1551.6 & 99.17 & $1.5515 e-10$ & -32.6936 \\
1552.4 & 99.17 & $1.5523 e-10$ & -32.6992 \\
1553.2 & 99.16 & $1.5531 e-10$ & -32.7052 \\
1554 & 99.16 & $1.5539 e-10$ & -32.7117 \\
1554.8 & 99.16 & $1.5547 e-10$ & -32.7187 \\
1555.6 & 99.16 & $1.5555 e-10$ & -32.7261 \\
1556.4 & 99.15 & $1.5563 e-10$ & -32.7339 \\
1557.2 & 99.15 & $1.5571 e-10$ & -32.7422 \\
1558 & 99.15 & $1.5579 e-10$ & -32.7509 \\
\hline
\end{tabular}

Figure 15 shows the behavior of the gain of the developed model with FBG and EDFA and without FBG and EDFA, based on different wavelengths. Results prove that, with FBG and EDFA we obtain better Gain as compared to the developed model without FBG and EDFA. It can also be 
TABLE 3: Wavelengths corresponding to different values of reflectivity peaks before and after FBG.

\begin{tabular}{lccccccccc}
\hline & Before FBG & 1546 & 1546.8 & 1547.6 & 1548.4 & 1549.2 & 1550 & 1550.8 & 1551.6 \\
Wavelengths (nm) & After FBG & 1546 & 1546.81 & 1547.62 & 1548.4 & 1549.19 & 1550 & 1550.79 & 1551.61 \\
& Before FBG & 1552.4 & 1553.2 & 1554 & 1554.8 & 1555.6 & 1556.4 & 1557.2 & 1558 \\
& After FBG & 1552.41 & 1553.22 & 1554 & 1554.8 & 1555.59 & 1556.4 & 1557.21 & 1558 \\
\hline
\end{tabular}

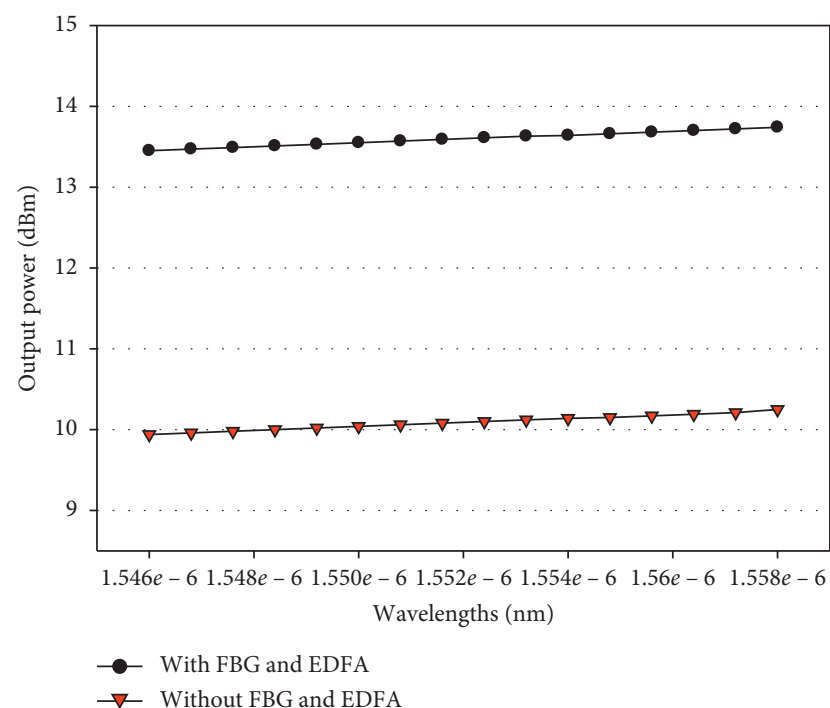

FIgURE 14: Output variation at different values of wavelengths.

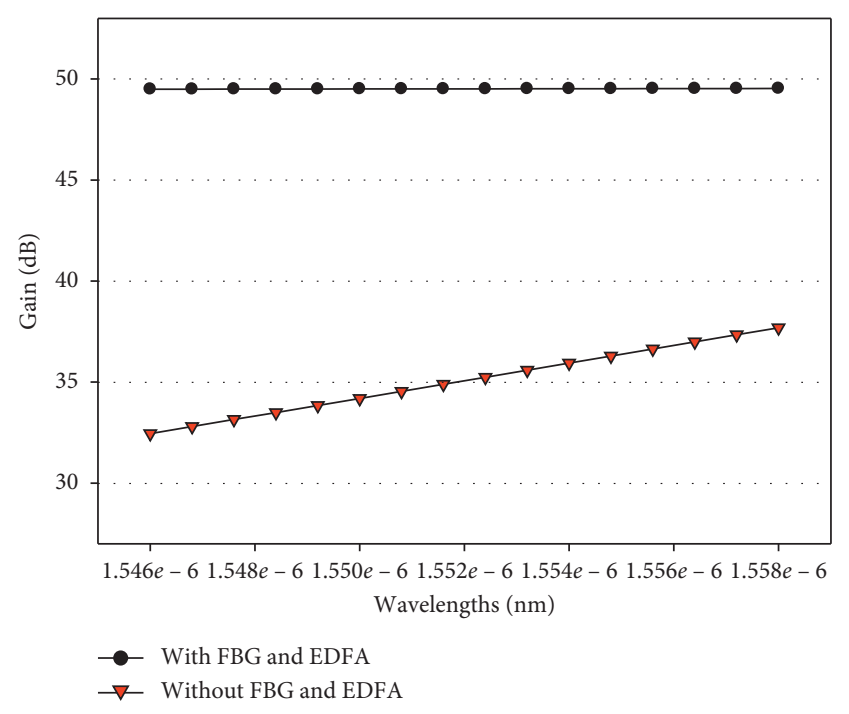

FIGURE 15: Gain variation at different values of wavelengths.

noticed that the gain of the developed method with FBG and EDFA remains almost equal in the six wavelengths, which can be considered as an added value of our developed optical network [3].

\section{Conclusion}

In this paper, a new optical network model with the introduction of the FBG and EDFA has been developed to determine the gain of the optical transmission network and to study the performance of an optical transmission system of 16 channels in terms of nonlinear and dispersion parameters. The SSFM method for numerical modeling was applied to solve the nonlinear Schrödinger equations to decrease the linear and nonlinear effects. CMT and TMM were also applied to obtain optimized reflection spectrum with high reflectivity, small FWHM, and an efficient side lobe suppression. To solve the challenge of compensating the chromatic dispersion and reduce the nonlinear effects, a simple technique has been applied; this technique consists on linking the FBG and EDFA to deal the output power and the gain of the optical transmission system. Simulation results show that the proposed method results provide better responses in comparison with other works, which demonstrates further the efficiency of the developed technique.

\section{Data Availability}

This work did not receive specific funding but is performed as part of the employment of the authors. The employer is the University of Abdelmalek Essaâdi, Tetuan-Morocco. The SIG-Laboratory can be contacted at this e-mail address: yassin.chkouri@gmail.com.

\section{Conflicts of Interest}

The authors declare that there are no conflicts of interest regarding the publication of this paper.

\section{References}

[1] R. Ramaswami, K. N. Sivarajan, and G. H. Sasaki, Optical Networks: A Practical Perspective, Elsevier, Amsterdam, Netherlands, 3rd edition, 2010.

[2] N. Antoniades, G. Ellinas, and L. Roudas, WDM Systems and Networks: Modeling, Simulation, Design and Engineering, SpringerScience+Business Media, Berlin, Germany, 2012.

[3] M. Chakkour, O. Aghzout, B. Aitahmed, F. Chaoui, A. V. Alejos, and M. El yakhloufi, "Gain flatness and noise figure optimization of $C$-band EDFA in 16-channels WDM system using FBG and GFF," International Journal of Electrical and Computer Engineering (IJECE), vol. 7, no. 1, p. 289.

[4] S. P. Singh, S. Iyer, S. Kar, and V. K. Jain, "Study on mitigation of transmission impairments and issues and challenges with PLAI-RWA in optical WDM networks," Journal of Optical Communication, vol. 33, no. 2, pp. 83-101, 2012.

[5] D. Malik, K. Pahwa, and A. Wason, "Performance optimization of SOA, EDFA, Raman and hybrid optical amplifiers in WDM Network with reduced channel spacing of $50 \mathrm{GHz}$," Optik, vol. 127, no. 23, pp. 11131-11137, 2016.

[6] A. H. Beshr, "Study of ASE noise power, noise figure and quantum conversion efficiency for wide-band EDFA," Optik, vol. 126, no. 23, pp. 3492-3495, 2015.

[7] S. Semmalar and S. Malarkkan, "output signal power analysis in erbium-doped fiber amplifier with pump power and length 
variation using various pumping techniques," ISRN Electronics, vol. 2013, no. 6, Article ID 312707, 2013.

[8] P. A. Govind, Nonlinear Fiber Optics, Academic Press, Cambridge, MA, USA, 3rd edition, 1995.

[9] S. Iyer and S. P. Singh, "Theoretical evaluation of combined nonlinearities and ASE noise penalties in optical star WDM networks based on ITU-T conforming optical fibers," The Institution of Electronics and Telecommunication Engineers (IETE) Journal of Research, vol. 58, no. 6, pp. 482-492, 2012.

[10] S. P. Singh and S. Iyer, "Impact of SRS and FWM on performance of optical star WDM networks," in Proceedings of the IEEE Symposium on Photonics and Optoelectronics (SOPO 2011), pp. 1-4, Wuhan, China, May 2011.

[11] F. Chaoui, O. Aghzout, M. Chakkour, and M. El Yakhloufi, Theoretical Approach to Evaluate Fiber Bragg Grating Sensor 4 Apodization Performance using MATLAB-Based New Code, Hindawi, London, UK, 2016.

[12] S. P. Singh and S. Iyer, "Effect of SRS and FWM on performance of optical star WDM networks: a deterministic approach," in Proceedings of the IEEE 7th International Conference on Wireless and Optical Communications Networks (WOCN 2010), pp. 1-4, Colombo, Srilanka, September 2010.

[13] J. Sajgalikova, M. Dado, and J. Litvik, "Comparison of numerical modelling of degradation mechanisms in single mode optical fiber using MATLAB and VPI photonics," Advances in Electrical and Electronic Engineering, vol. 13, no. 3, 2015.

[14] A. H. M. Huseina and F. I. El-Nahal, "Optimizing the EDFA gain for WDM lightwave system with temperature dependency," Optik, vol. 123, no. 7, 2011.

[15] A. Bindal and S. Singh, "Dispersion analysis of fiber Raman amplifier for WDM system," Optics \& Laser Technology, vol. 58, pp. 20-25, 2014.

[16] M. Chakkour, O. Aghzout, B. Ait Ahmed, F. Chaoui, and M. El Yakhloufi, "Chromatic dispersion compensation effect performance enhancements using FBG and EDFA-wavelength division multiplexing optical transmission system," International Journal of Optics, vol. 2017, Article ID 6428972 , 8 pages, 2017. 